\title{
A MEMETIC ALGORITHM WITH VARIABLE LENGTH CHROMOSOME FOR ROBOT PATH PLANNING UNDER DYNAMIC ENVIRONMENTS
}

\author{
Jianjun $\mathrm{Ni},{ }^{*, * *}$ Kang Wang, ${ }^{*}$ Qingyun Cao, ${ }^{*}$ Zubair Khan, ${ }^{*}$ and Xinnan Fan,**
}

\begin{abstract}
There are some shortcomings of the traditional methods for robot path planning, such as the local minimum problem and the low convergence speed in the genetic algorithm (GA)-based methods. In this paper, an improved memetic algorithm is proposed for robot path planning. In the global search process of the proposed memetic algorithm, a GA with variable length chromosome based on improved two-point crossover and bacterial mutation is used to avoid the local optimum problem. And a search method which combines a neighbouring local search and a disorder strategy is used in the local search process of the proposed approach to speed up the convergence procedure. Furthermore, a dynamic module is added in the proposed approach to deal with the path planning in dynamic environments. Finally, some simulation and real robot experiments are carried out and the experimental results show the efficiency and the effectiveness of the proposed algorithm in the path planning of mobile robots.
\end{abstract}

\section{Key Words}

Mobile robot, path planning, memetic algorithm, variable length chromosome

\section{Introduction}

Path planning is one of the hot spots in the field of mobile robots, which means finding an optimal or near optimal collision-free path from the starting position to the target position in an environment with obstacles [1-4]. There have been various methods used for robot path planning, and the classical methods include artificial potential field methods [5], grid map representation methods [6] and

* College of IOT Engineering, Hohai University, Changzhou 213022, People's Republic of China; e-mail: \{njjhhuc, engr. zubairkhan13\}@gmail.com, \{wangk9294, qycao666, fanxn519\}@ 163.com

** Jiangsu Universities and Colleges Key Laboratory of Special Robot Technology, Hohai University, Changzhou 213022, People's Republic of China

Recommended by Dr. Daqi Zhu

(DOI: 10.2316/Journal.206.2017.4.206-4998) visibility graph methods [7]. However, there are some limitations of those traditional methods, such as the local minimum problem in the traditional artificial potential field methods and the complex computations in the grid map-based methods. Recently, with the development of the bionic technology, the bio-inspired intelligent algorithms have been widely used in the path planning of mobile robots [8]. The traditional bio-inspired algorithms have been proved to have some global search capability and parallelism, but each method has its own limitations. For example, the genetic algorithm (GA) has the disadvantage of slow convergence rate, large search space and premature convergence [9]; and the computation of the general neural network-based methods is complex and it needs a learning process, which sometimes cannot meet the requirements of real-time applications [10].

To deal with the problems of those traditional bioinspired intelligent algorithms in robot path planning, some improvements have been proposed. For example, Montiel et al. [11] presented a novel bacterial potential field method for path planning; Ni et al. [12] proposed a Q-learning algorithm based on a bio-inspired neural network for robot path planning; Xue and Liu [13] presented an improved particle swarm optimization (PSO) algorithm for mobile robot path planning in complex indoor environment; He and Dai [14] presented an improved niched GA for stealth corridor real-time path planning for heterogeneous unmanned aerial vehicles in three-dimensional (3D) dynamic environments. These methods introduced above have some advantages. However, much of the research is focused on the parameters optimization, and few of them considered the optimization problem of the whole process for robot path planning.

Recently, the memetic algorithm has been proposed to deal with the path planning problem, which is a novel bio-inspired intelligent algorithm proposed to simulate the culture evolution of human society [15], [16]. The memetic algorithm is a combination of the swarm-based evolutionary global search and the individual-based heuristic local search. However, there are some limitations in the global and the local search process in the memetic algorithm by 
using general optimization algorithms. In addition, there are other problems that should be solved when the memetic algorithm is used for robot path planning, such as the environment modelling and the fitness function design. For example, the memetic algorithm-based path planning method in [15] used a fixed length chromosome, which is not suitable for path planning in complex environment, because the number of nodes in the path cannot be known previously. To deal with these problems, an improved memetic algorithm (IMA) is proposed in this paper. In the proposed approach, an adaptive strategy is adopted to find a proper length of the chromosome in the memetic algorithm. An improved two-point crossover method is used to improve the performance of global search. And a neighbouring search combined with a disorder strategy is used to accelerate the speed of local search. In addition, a concept of risk level is introduced into the fitness function to improve the safety and effectiveness of the proposed path planning approach. To deal with the problem of path planning in dynamic environments, a dynamic module is added in the proposed approach. Finally, some simulation and real robot experiments were conducted. The results show that both the efficiency and the convergency speed of the proposed approach are increased obviously.

This paper is organized as follows. Section 2 presents the proposed improved memetic algorithm. The simulation experiments for mobile robot path planning are given in Section 3. Section 4 discusses the performance of the proposed approach in detail. In Section 5, the proposed approach is validated in a real robot. Finally, the conclusions are given in Section 6 .

\section{The Improved Memetic Algorithm}

In this paper, the robot path planning based on the memetic algorithm is studied [17], and an improved memetic algorithm is proposed for robot path planning. There are some significant differences between the literature and the proposed approach in this paper. For example, the strategy of the adaptive changing length of the chromosome and the fitness function of the proposed approach are different from those in the literature [15]; and the whole work procedure and the disorder strategy are different from that in the literature [18]. Here only the improvements of the proposed approach are introduced in detail as follows.

\subsection{Environment Modelling}

In this paper, the grid map is used to model the environment for robot path planning [6]. An example of this environment is shown in Fig. 1, where the obstacles are denoted by grey grids and the potential path of the robot is constructed by the grid without obstacles. In the proposed memetic algorithm, the chromosome represents a path from the start point to the goal point. To easily realize the encoding for the chromosome, the location of each point is denoted by a unique integer number, then one of the chromosomes in Fig. 1 is encoded as $(1,3,21$, $22,34,36)$.

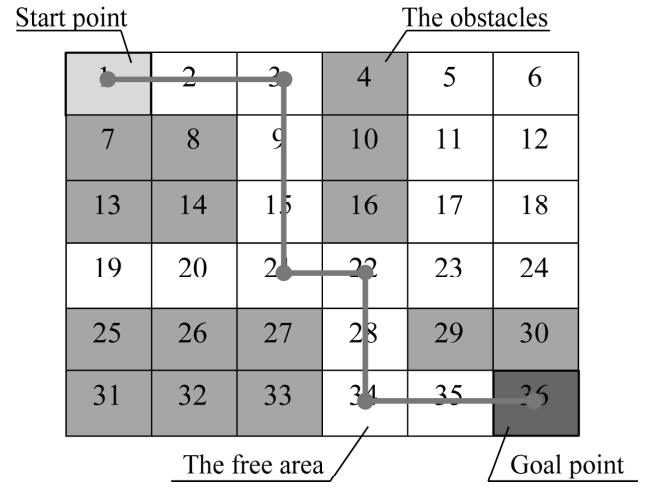

Figure 1. An example of the environment modelled by the grid map.

In the memetic algorithm, the number of the points $N$ of the path, namely the length of the chromosome, is a very important parameter. The larger the number of the points, the more the computational time is needed. However, if the number of the points is too small, the path planning task may get failed. So, it is a difficult task to find a proper length of the chromosome for the memetic algorithm in the task of robot path planning. In this paper, an adaptive function is used to determine the number of the points $N$. At first, an initial value of $N$ is calculated based on the experience and the condition of environment:

$$
N=\alpha+\operatorname{round}\left(\frac{N_{O}}{N_{G}}\right) * \beta
$$

where $\alpha$ is a constant number, which is decided by experience; $N_{O}$ and $N_{G}$ denote the number of the obstacle grids and the total grids in the map, respectively; $\beta$ is a coefficient to decide the effect of the obstacles; round $\{\cdot\}$ is the rounding function. To further consider the effect of the population information during the optimization process, the initial value of $N$ is changed dynamically by the rule as follows:

If condition $1 \&$ condition $2 \&$ condition 3 then $N=N+2$

where "condition1" is that $N_{\text {steps }}>100$ and $N_{\text {steps }}$ is the number of the iteration steps in current value of $N$; "condition2" is that $f_{\min }<\tau$ and $\tau$ is a threshold which is set as 90 in this paper; and "condition3" is that $\left(f_{\max }-f_{\text {ave }}\right) / f_{\max }<\varepsilon$ within 10 steps continuously and $\varepsilon$ is a constant number which is set as 0.1 in this paper, $f_{\max }, f_{\min }$ and $f_{\text {ave }}$ are the maximum fitness, the minimum fitness and the average fitness (see Section 2.2) in one iteration step.

\subsection{The Fitness Function}

The fitness function is a key part in all of the evolution optimization algorithms. In the path planning task of mobile robot, two most important evaluation indexes are the path length and the safety of the robot. Recently, the energy consumption is also considered into the path planning for mobile robot. So in this paper, a comprehensive fitness 
function is proposed, which includes three parts and is defined as follows:

$$
f_{o}(p)=w_{d} d(p)+w_{o} o(p)+w_{s} s(p)
$$

where $d(p)$ is the path length; $o(p)$ is the risk level of the path; $s(p)$ is the smoothness of the path; and the parameters $w_{d}, w_{o}, w_{s}$ are the weights for $d(p), o(p)$ and $s(p)$.

In this paper, $d(p)$ is calculated by

$$
d(p)=\sum_{i=1}^{N} \operatorname{Dist}\left(z_{i}, z_{i+1}\right)
$$

where $\left(z_{i}, z_{i+1}\right)$ denotes the end points of the line segment $l_{i}$, and function Dist $(\cdot)$ denotes the length of the line segments $l_{i}$ in the path $p$. The risk level $o(p)$ is used to evaluate the safety of the path for the robot, which can be calculated by

$$
o(p)=\left\{\begin{array}{cl}
n_{o} \times a+b & \text { if it is an infeasible path } \\
0 & \text { if it is a feasible path }
\end{array}\right.
$$

where $n_{o}$ represents the sum of the number of the obstacles the robot collided with or passed through on the path $p ; a$ is a penalty coefficient for the path $p$; and $b$ is an adjustment parameter.

During the movement, the energy consumption is high and the stability of the robot is low if the path is not smooth. So, a concept of smoothness is introduced into the fitness function [18], which can be calculated by

$$
s(p)=\frac{c}{\sum_{i=1}^{N-1} \theta\left(l_{i}, l_{i+1}\right)}
$$

where $\theta\left(l_{i}, l_{i+1}\right)$ denotes the angle between line segments $l_{i}$ and $l_{i+1}$; and $c$ is a constant number.

\subsection{The Global Search}

In the global search of the memetic algorithm, more attention should be paid to the effectiveness issue. In the general genetic algorithm (GGA)-based search methods, the search efficiency will be low if the solution space is very large. To deal with this problem, an improved GA is used in the global search of the proposed memetic algorithm. In this paper, the selection strategy is based on both the roulette wheel selection and the elite selection methods (see [18] and [19] for details), and the mutation operation is based on the bacterial mutation operator, to avoid the local optimum problem (see [15] and [20] for details). Here only an improved crossover operator is introduced.

The traditional two-point crossover is unable to overcome the local minimum problem for its less disruptive to the chromosome [21]. To deal with this problem, an improved two-point crossover is proposed which is based on the individual difference $D_{I}$ in the population, which is calculated by:

$$
D_{I}=\frac{f_{\max }-f_{\text {ave }}}{f_{\max }} \in[0,1]
$$

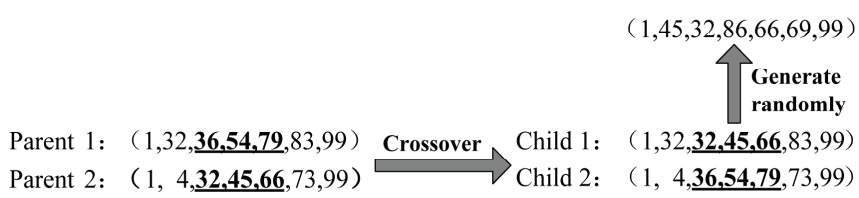

(a)

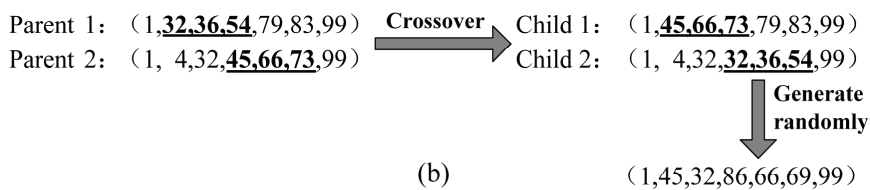

Figure 2. An example of the two-point crossover in the global search: (a) the traditional two-point crossover and (b) the unfixed site two-point crossover.

When $D_{I} \geq 0.5$, the traditional two-point crossover method is used (see Fig. 2(a)). Otherwise, an unfixed site two-point crossover is applied, which is performed by exchanging the gene segments of parents between two unfixed sites (see Fig. 2(b)). Note that if an offspring contains more than one of the same nodes, then it should be deleted and a new path should be initialized randomly.

\subsection{The Local Search}

The local search is a very important part of the memetic algorithm. In this study, a disorder strategy and a neighbouring local search are used in the local search process to accelerate the evolutionary process. The detail process of the proposed disorder strategy is as follows: At first, the chromosome with $N-1$ line segments is disordered and rearranged, where the first and the last point in the chromosome must be kept unchanged. This process will be repeated $f$ times and $N_{\text {local }}$ new chromosomes are obtained. Then, the chromosome with the best fitness is selected from all the $N_{\text {local }}$ chromosomes and the original chromosome.

After the disorder operator, a neighbouring local search is adopted [22]. To the chromosome obtained by the disorder strategy, the final point of the line segment which is intersected by the obstacles will be replaced by its neighbouring gene one by one (in this paper, each gene has eight neighbouring genes and the gene occupied by the obstacles will be wiped out directly). And the chromosome with the best fitness will be selected from all the new chromosomes by this replacement operator. When all the $N-1$ line segments of the chromosome are conducted by this local search, the final chromosome is used as the new chromosome.

\subsection{The Dynamic Module}

In the traditional GA-based path planning method, the environments are always assumed to be static. But as we know, the environment where the robot works is often dynamic [23], [24], because there are often many moving objects, such as human beings and pets. To deal with this problem, a module for dynamic environment is added in 


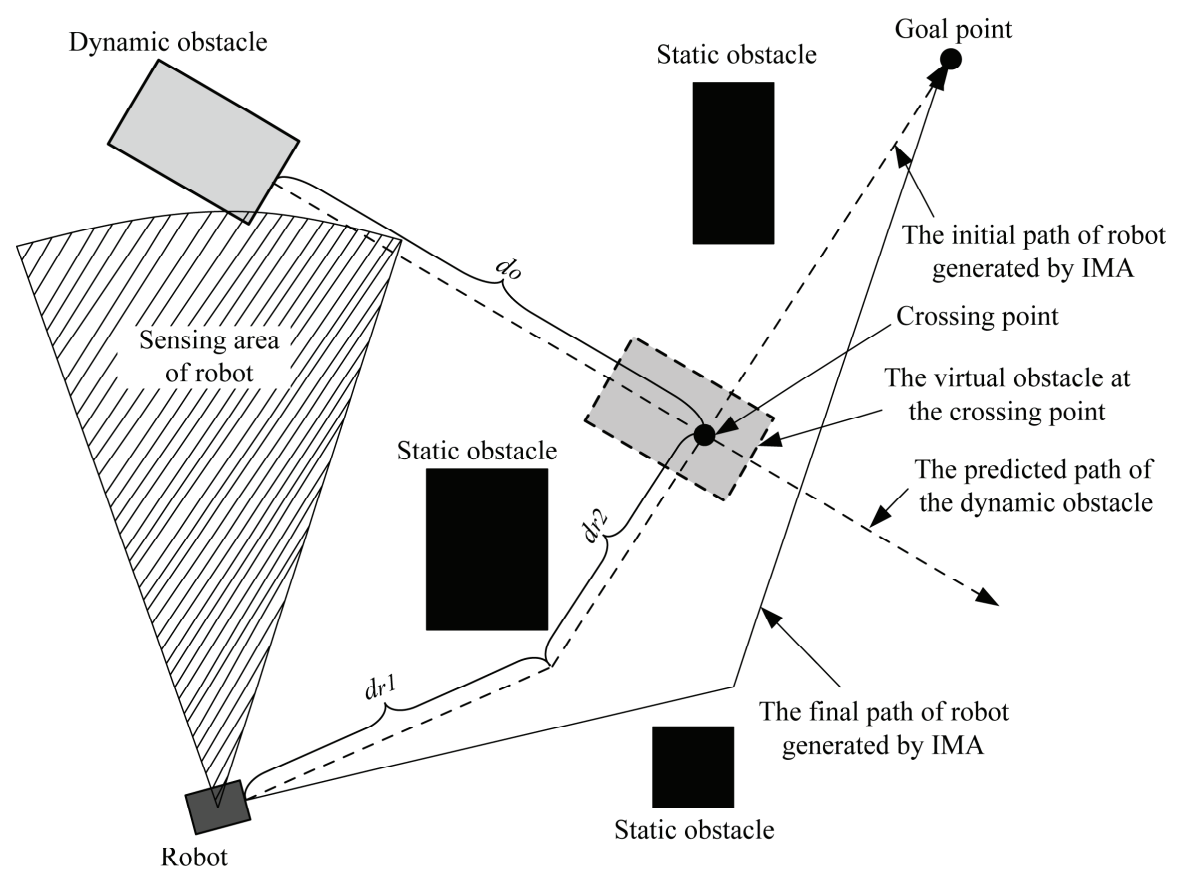

Figure 3. The sketch map of the dynamic module to deal with the dynamic obstacle.

the proposed approach. The basic idea of the proposed dynamic module is to generate a virtual static obstacle for the dynamic obstacle, which is based on the movement parameters of the moving obstacle. The basic work flow of the dynamic module is as follows [25], [26]: (1) when a moving obstacle is observed, the trajectory of this moving obstacle is predicted based on its movement parameters and (2) if the robot collides with the moving obstacle, the path of the robot will be planned again based on the trajectory and the direction of the moving obstacle. A schematic diagram of this working process for robot path planning in dynamic environment is shown in Fig. 3.

Remark. Because the proposed method is based on the observation of the moving objects, the robot cannot deal with some challenging cases, such as irregular and rapid motion of the moving objects.

\section{Simulation Experimental Studies}

To test the performance of the path planning based on the proposed memetic algorithm, some experiments are conducted for various situations. The parameters of the proposed approach in all the experiments are the same (see Table 1), where the experimental environment is $20 * 20$ $\left(\mathrm{m}^{2}\right)$. To illustrate the performance of the IMA in this paper, it is compared with the GGA and the general memetic algorithm (GMA). All the same parameters of the three methods are set to the same values for the comparability, such as the maximum iteration number, the crossover probability and so on. In GGA and GMA, the selection operator is the same as IMA, the crossover operator is traditional two-point crossover and the mutation operator is random mutation (the probability of mutation is 0.15 in GGA and GMA). In each experiment, the number of points $N$ in GGA and GMA is the same and set by the experience (where $N$ are set as 12, 14, 10 in the next three experiments). In these experiments, the parameters $w_{d}, w_{o}, w_{s}$ are the same, namely $w_{d}=\frac{1}{3}, w_{o}=\frac{1}{3}$ and $w_{d}=\frac{1}{3}$. Because there are some random operations

Table 1

Parameters of the Proposed Approach for Robot Path Planning

\begin{tabular}{|l|r|l|}
\hline Parameters & Value & Remarks \\
\hline$P_{C}$ & 0.9 & The crossover probability \\
\hline$P_{M}$ & 0.8 & The mutation probability \\
\hline$P_{L}$ & 0.3 & The local search probability \\
\hline$S_{P}$ & 60 & The population size \\
\hline$M_{G}$ & 200 & The maximum generations \\
\hline$a$ & 50 & The penalty coefficient in \\
\hline & & calculating $o(p)$ \\
\hline$b$ & 30 & The adjustment parameter in \\
\hline & & calculating $o(p)$ \\
\hline$c$ & 800 & The constant number in \\
\hline & & calculating $s(p)$ \\
\hline$f$ & 10 & The number of the repetitions \\
\hline & & for the disorder strategy \\
\hline$\alpha$ & 6 & The parameter in calculating $N$ \\
\hline$\beta$ & 10 & The parameter in calculating $N$ \\
\hline
\end{tabular}



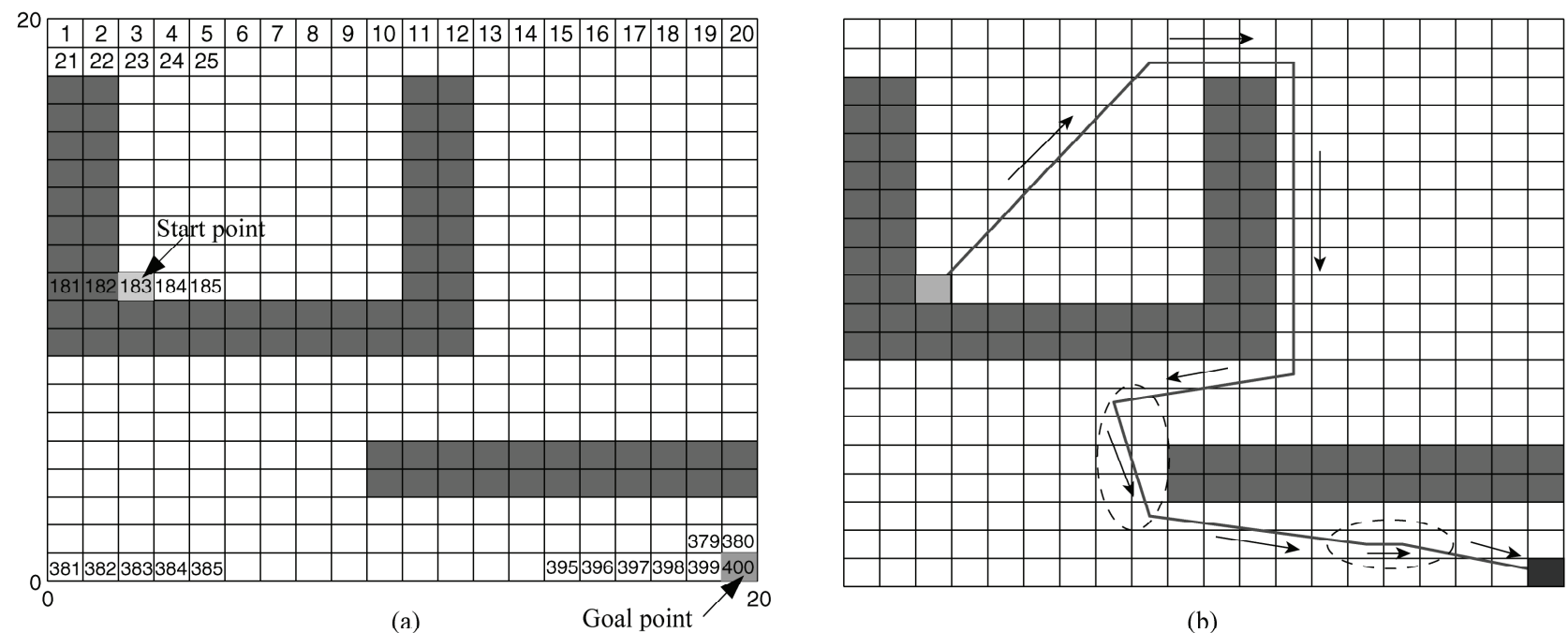

(a)

Goal point

(b)
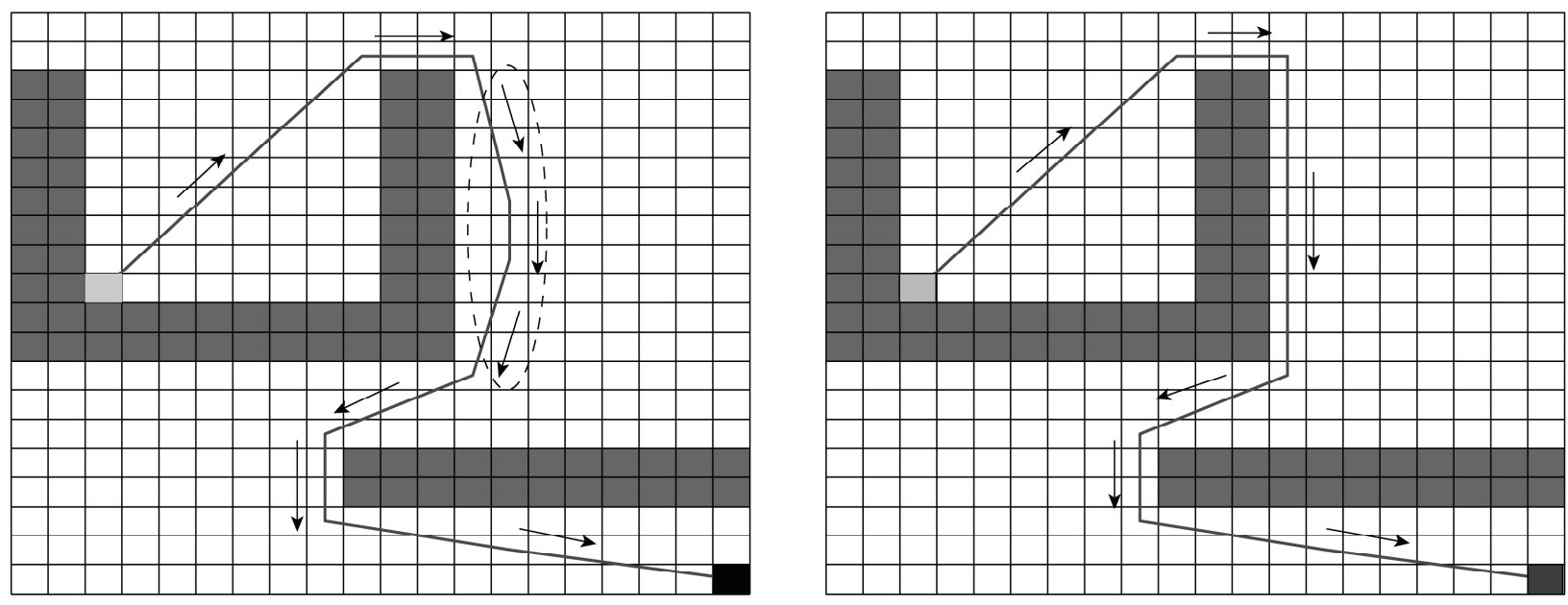

(c)

(d)

Figure 4. The path planning results of three methods under simple environment: (a) the initial environment of the experiment; (b) based on the GGA method; (c) based on the GMA method; and (d) based on the IMA method.

in these three methods, each experiment is conducted 10 times to remove the effects of the randomness. For simplification without losing generality, the robot is assumed to be a point without having any shapes.

\subsection{Under Static Environment}

To prove the performance of the proposed approach in robot path planning tasks under static environment, two experiments are conducted. One is under a simple environment and other one is under a complex environment.

\subsubsection{Under Simple Environment}

In this experiment, the environment is simple where the number of the obstacles is small and the goal point is easy to reach. The initial positions of the robot, the goal and the obstacles are shown in Fig. 4(a), where the start point of the robot is 183 , and the goal is located at the point 400 . The path planning results of the three methods are shown in Table 2 and Fig. 4(b)-(d).
The results show that all the three methods can complete the path planning task in this simple environment. However, the average path length and the total turning angles of the robot based on both the GGA and the GMA method are larger than the proposed IMA approach (see Fig. 4 and Table 2). The results mean that the proposed method can complete the path planning task effectively and can generate an optimal path that consumes less energy for the robot in this situation. In addition, the number of points in the path obtained by the IMA approach is 8 in this experiment, which is smaller than the GGA and the GMA method and can decrease the computation time of the path planning.

\subsubsection{Under Complex Environment}

In this experiment, the environment is more complex than the previous one, where the obstacles are of various shapes and the goal is located at a position difficult to reach. Figure 5(a) shows the initial positions of the robot, the goal and the obstacles, where the start position of the robot is 1 , 
Table 2

The Results of the Path Planning Task in Simple Environment

\begin{tabular}{|l|c|c|c|c|}
\hline $\begin{array}{l}\text { The path } \\
\text { planning method }\end{array}$ & $\begin{array}{c}\text { The average length } \\
\text { of the path }(\mathrm{m})\end{array}$ & $\begin{array}{c}\text { The deviation } \\
\text { of the path length }\end{array}$ & $\begin{array}{c}\text { The average total turning } \\
\text { angle of the robot }\left(^{\circ}\right)\end{array}$ & $\begin{array}{c}\text { The failed } \\
\text { times }\end{array}$ \\
\hline GGA & 46.79 & 4.12 & 426.11 & 0 \\
\hline GMA & 45.36 & 2.09 & 355.77 & 0 \\
\hline IMA & 43.78 & 0.54 & 346.21 & 0 \\
\hline
\end{tabular}
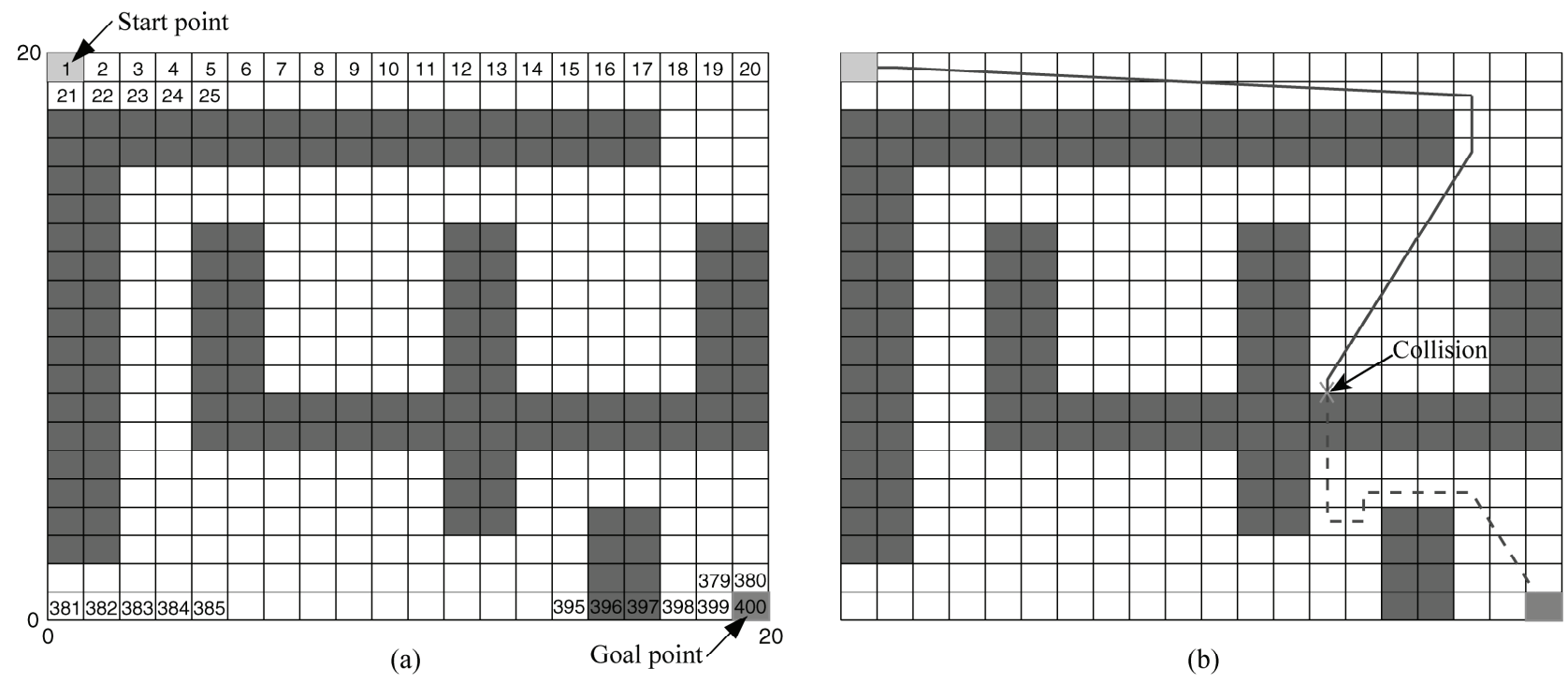

(a)

(b)

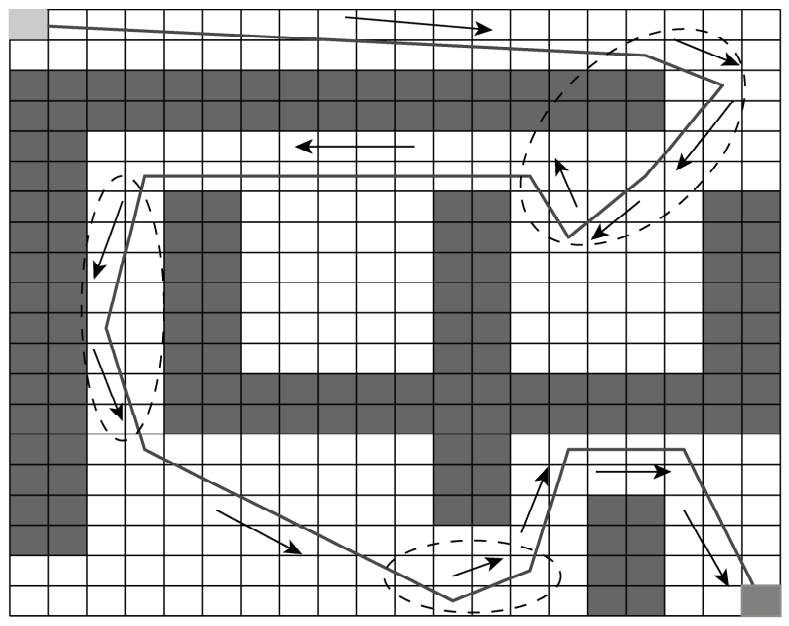

(c)

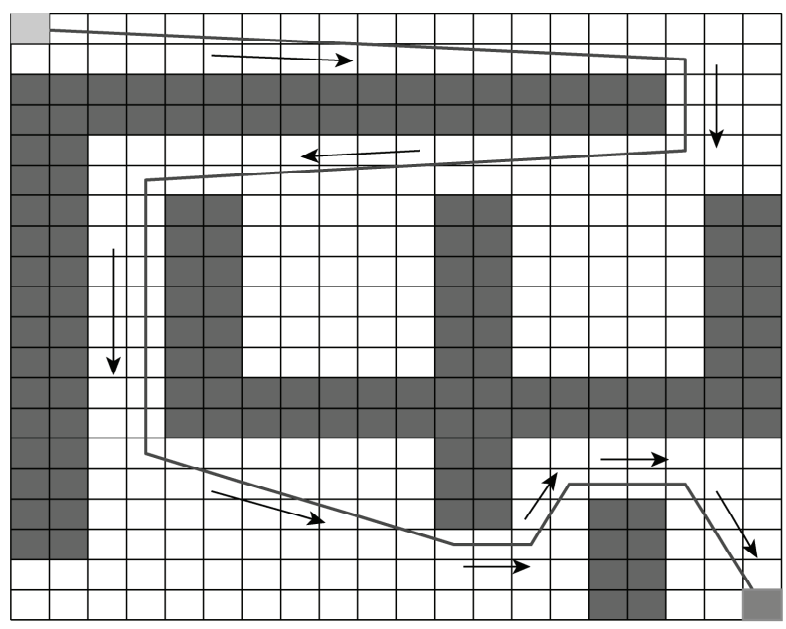

(d)

Figure 5. The path planning results of three methods under complex environment: (a) the initial environment of the experiment; (b) based on the GGA method; (c) based on the GMA method; and (d) based on the IMA method.

and the position of the goal is 400 . The experimental results are shown in Fig. 5 and Table 3.

The results in Fig. 5 show that the proposed approach can generate an optimal path for the robot under this complex environment. However, the general GGA method cannot find a feasible path for robot in limited iteration steps under this complex environment. Although the general GMA method can find some feasible path for robot, but the smoothness of the path generated by the general GMA method is less than that of the proposed approach and the length of the path generated by the GMA method is bigger than that of the proposed approach (see Fig. 5 and Table 3). The main reason is that the global and local search in the proposed memetic algorithm is more efficient than the general memetic algorithm, and the proposed approach has a variable length chromosome, 
Table 3

The Results of the Path Planning Task in Complex Environment

\begin{tabular}{|l|c|c|c|c|}
\hline $\begin{array}{l}\text { The path } \\
\text { planning method }\end{array}$ & $\begin{array}{c}\text { The average length } \\
\text { of the path }(\mathrm{m})\end{array}$ & $\begin{array}{c}\text { The deviation } \\
\text { of the path length }\end{array}$ & $\begin{array}{c}\text { The average total turning } \\
\text { angle of the robot }\left(^{\circ}\right)\end{array}$ & $\begin{array}{c}\text { The failed } \\
\text { times }\end{array}$ \\
\hline GGA & $/$ & $/$ & $/$ & 10 \\
\hline GMA & 71.14 & 4.40 & 704.81 & 5 \\
\hline IMA & 64.12 & 0.66 & 539.25 & 0 \\
\hline
\end{tabular}

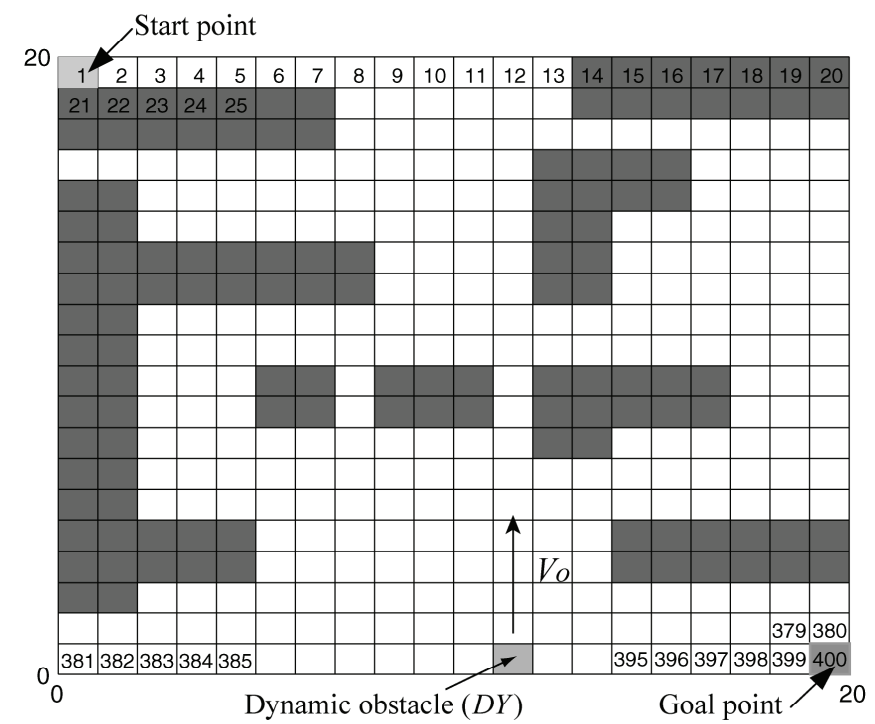

(a)

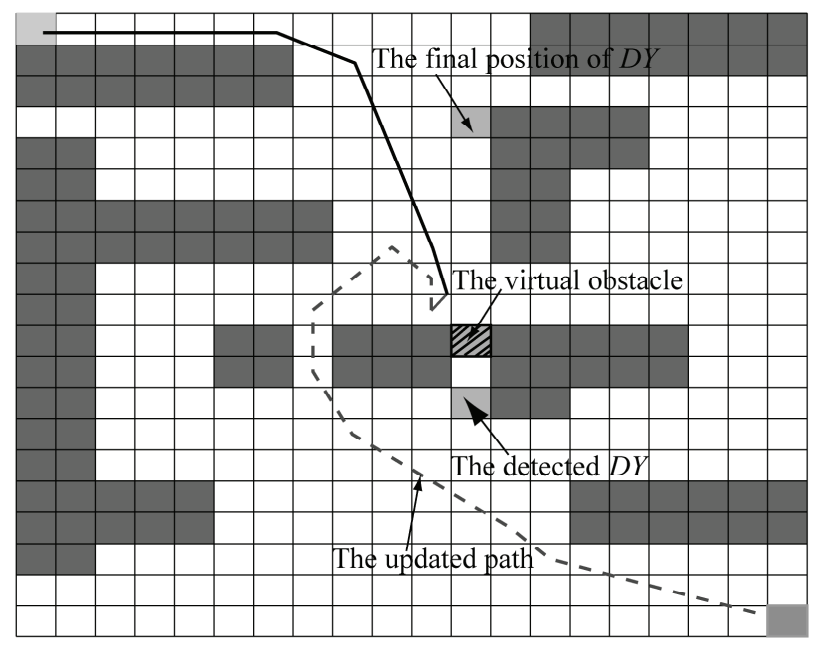

(c)

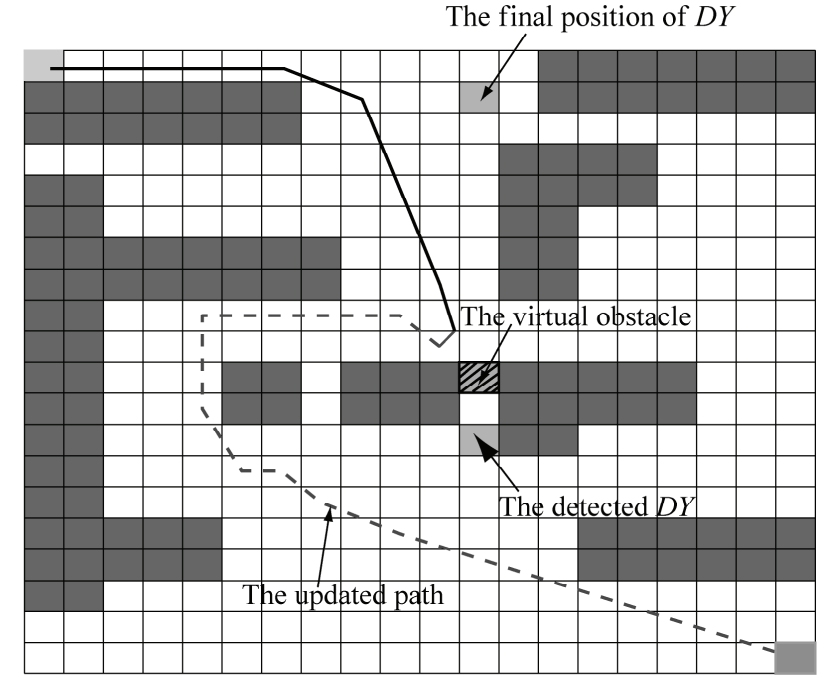

(b)

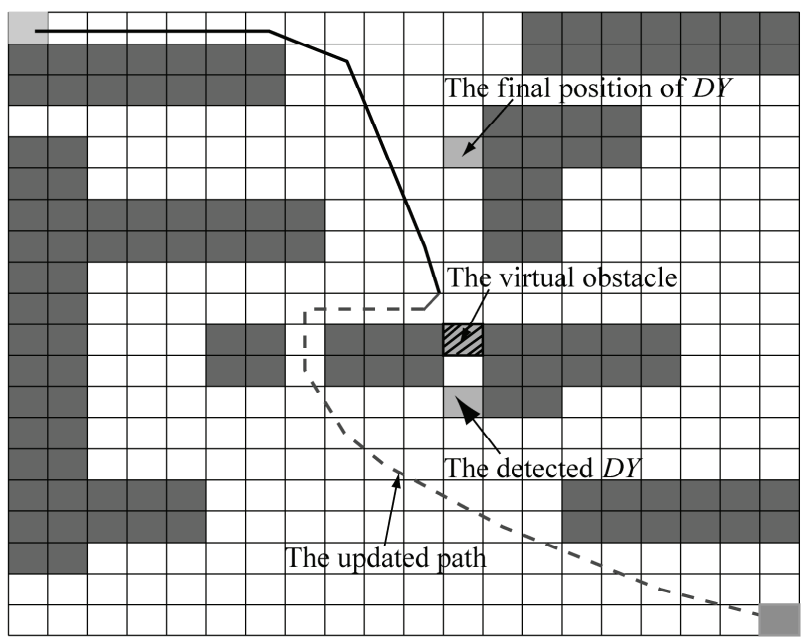

(d)

Figure 6. The path planning results of three methods under dynamic environment: (a) the initial environment of the experiment; (b) based on the GGA method; (c) based on the GMA method; and (d) based on the IMA method.

having a value 10 in this experiment. And the proposed approach combines the global and local search very well. Furthermore, the efficiency of the global search in the general memetic algorithm based on the traditional two-point crossover and random mutation will decrease obviously in the path planning task under complex environment.

\subsection{Under Dynamic Environment}

To further test the performance of the proposed method in the dynamic environment, this experiment is conducted, where an obstacle will move. The initial environment is shown in Fig. 6(a), where the initial position of the robot 
Table 4

The Results of the Path Planning in Dynamic Environment

\begin{tabular}{|l|c|c|c|c|}
\hline $\begin{array}{l}\text { The path } \\
\text { planning method }\end{array}$ & $\begin{array}{c}\text { The average length } \\
\text { of the path }(\mathrm{m})\end{array}$ & $\begin{array}{c}\text { The deviation } \\
\text { of the path length }\end{array}$ & $\begin{array}{c}\text { The average total turning } \\
\text { angle of the robot }\left(^{\circ}\right)\end{array}$ & $\begin{array}{c}\text { The failed } \\
\text { times }\end{array}$ \\
\hline GGA & 43.74 & 9.69 & 536.30 & 1 \\
\hline GMA & 39.28 & 3.21 & 471.83 & 1 \\
\hline IMA & 36.72 & 0.50 & 342.06 & 0 \\
\hline
\end{tabular}

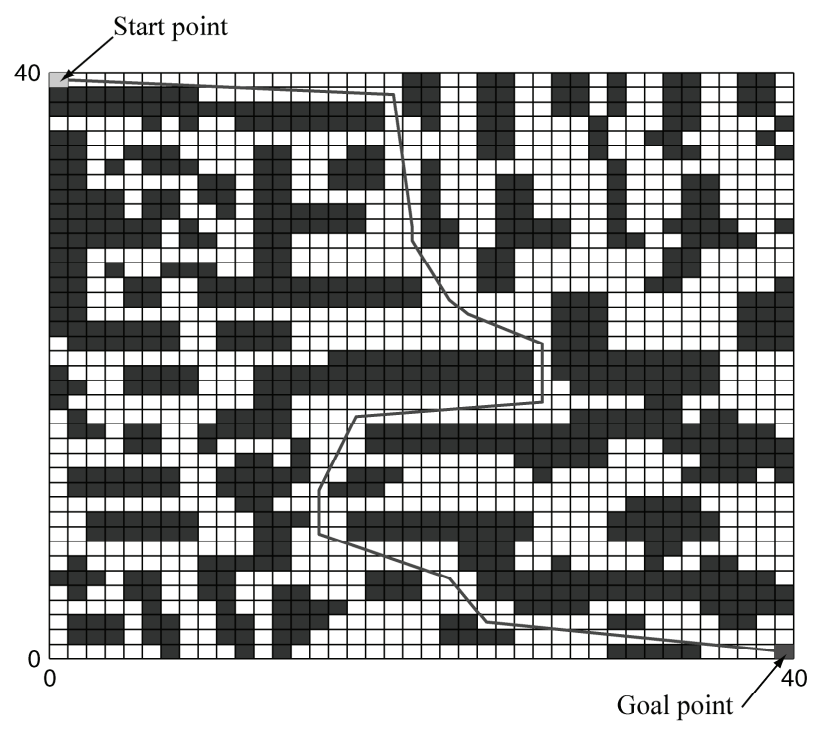

(a)

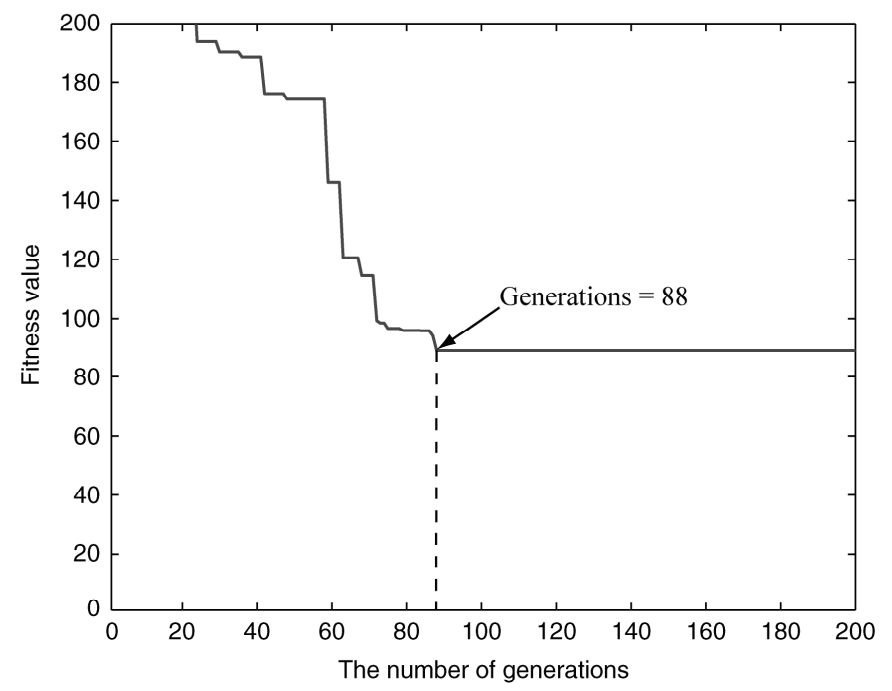

(b)

Figure 7. The path planning results based on the proposed approach under a large-scale environment.

and the goal are 1 and 400, respectively. The initial position of the moving obstacle is 392. The speed of the robot is set as $V_{r}=0.60 \mathrm{~m} / \mathrm{s}$. The moving obstacle will move from the bottom to the top of the environment at a speed $V_{o}=0.25 \mathrm{~m} / \mathrm{s}$. In this experiment, the work flow of the three methods to deal with the dynamic obstacles is the same, except the path planning approach is different. The path planning results are shown in Fig. 6 and Table 4.

The results show that the robot will plan an optimal path at first without knowing the dynamic obstacle and starts to move through this path. When the robot detects the dynamic obstacle, it will use the dynamic module to generate a new path for the robot to avoid the obstacle. The results in this dynamic experiment show that the proposed approach can generate a new path quickly and efficiently when a dynamic obstacle is detected (see Fig. 6 and Table 4), which is very important for robot path planning in dynamic environment. In addition, the deviation of the path length based on the proposed approach is less than that of the GGA and the GMA method, which shows that the proposed approach has better stability than the other two methods (see Table 4).

\section{Discussions}

The results of the simulation experiments in Section 3 show that the proposed approach can satisfy the path planning task under various situations. Some performances of the proposed approach are discussed in this section.

At first, the performance of the proposed approach in path planning under a large-scale environment is discussed. A simulation experiment is conducted, where the parameters of the proposed approach are the same as those in Section 3, except the environment is $40 * 40\left(\mathrm{~m}^{2}\right)$ and the distribution of the obstacles is more complex. The path planning results of the proposed approach are shown in Fig. 7. The results in Fig. 7 show that the proposed approach can deal with the path planning task under a largescale environment efficiently, and its performance does not decrease violently with the increasing size of the environment. The results of this experiment further show that the proposed approach can adapt to various complex environments very well, because the number of points in the path for the robot is computed adaptively, and both the global and local search ability of the proposed approach are increased.

To discuss the effects of the disorder strategy of the proposed approach, a comparison experiment is conducted, where the proposed approach is compared with a memetic algorithm without the disorder strategy. In this memetic algorithm, all the parameters and work flow are the same as the proposed approach, except the disorder strategy is removed. The second experiment in Section 3.1 is used as reference. The experimental results are shown in Fig. 8. 


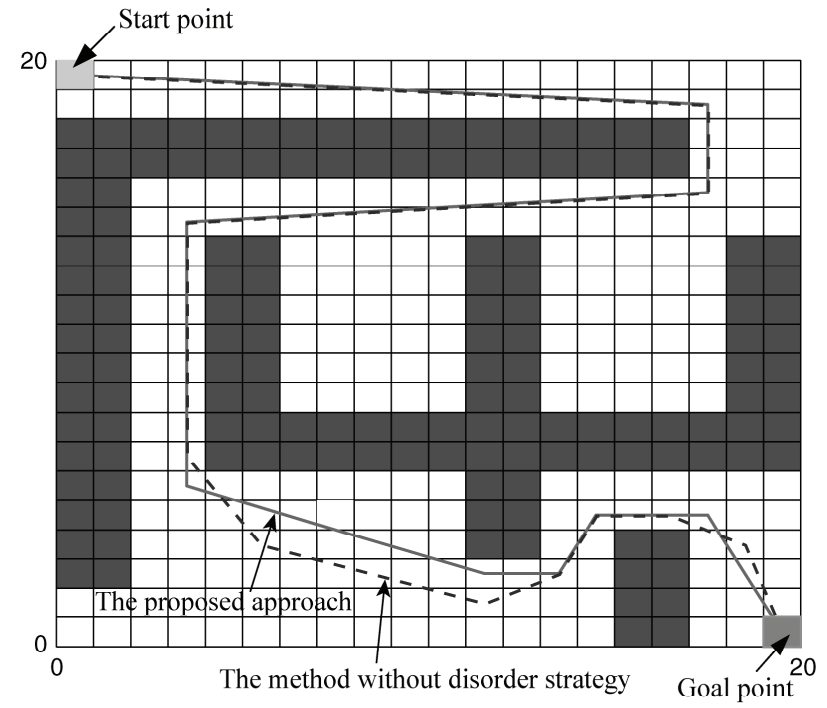

(a)

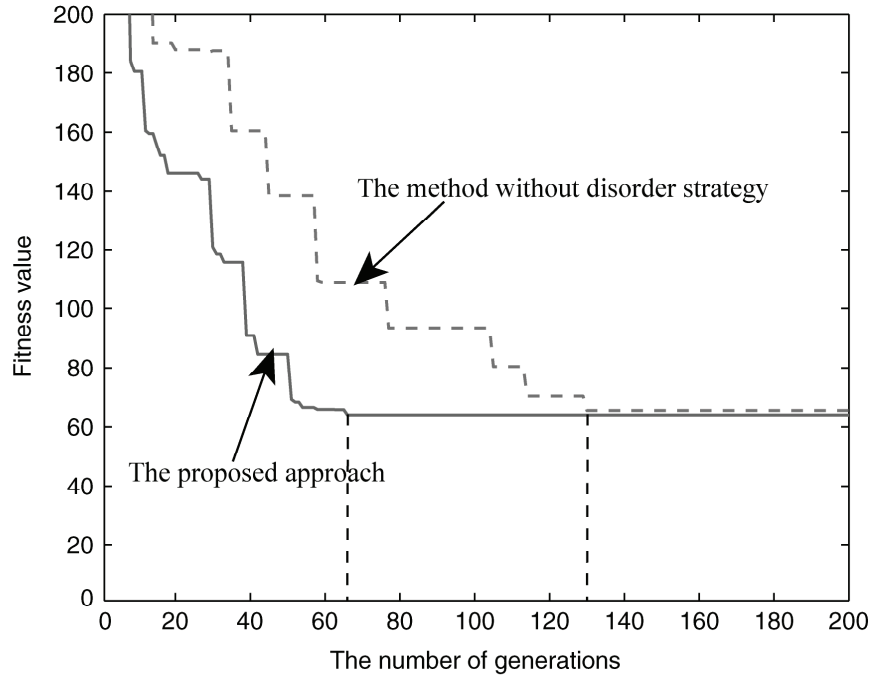

(b)

Figure 8. The path planning results based on the proposed approach and the method without the disorder strategy: (a) the generated paths based on the two methods and (b) the iteration processes of the two methods in this experiment.
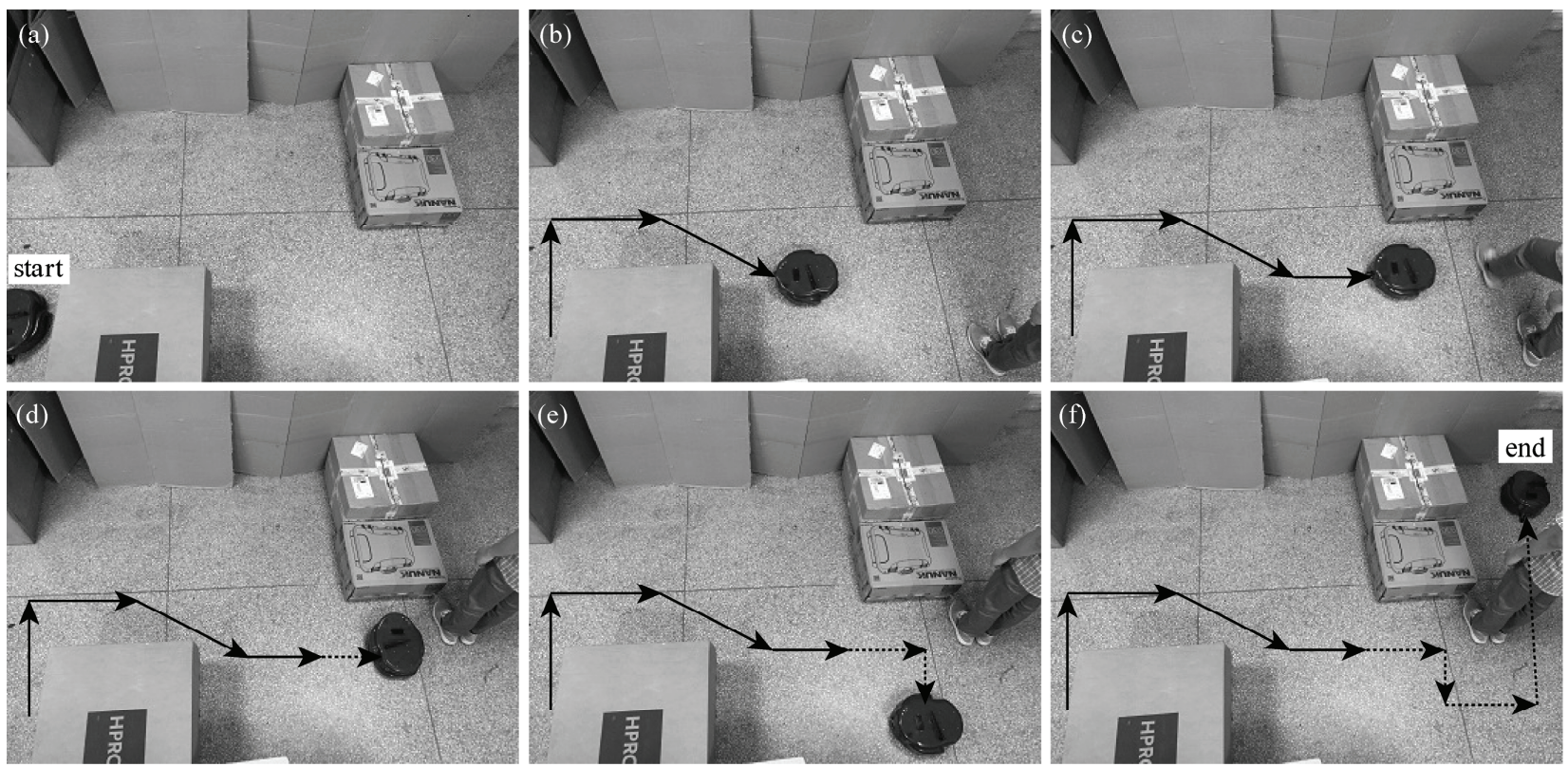

Figure 9. The real robot path planning experiment in a dynamic environment.

In this experiment, the memetic algorithm without the disorder strategy failed 3 times out of 10 . Obviously, the generated path and iteration process of the proposed approach are more optimal than the memetic algorithm without the disorder strategy (see Fig. 8).

\section{Application on a Real Robot}

To test the proposed approach in real robotic path planning, it is validated in the Amigo robot, which is the smallest member of the Pioneer family of mobile robot. The Amigo robot in this experiment is equipped with eight ultrasonic sensors to measure the distance between robot and the obstacles around it, and two encoders to calculate the moving distance of the robot. The results of the real robotic path planning experiment are shown in Fig. 9.

The results in Fig. 9 show that the Amigo robot can go through the static environment quickly and avoid the collision with obstacles automatically (see Fig. 9(a) and (b)). In this experiment, a human will suddenly appear in 
the working environment and get in the way of the robot, which will cause the robot to stop (see Fig. 9(c)), and a new path will be generated quickly and the robot can be navigated to the target safely (see the dashed line in Fig. 9). The path length and the total turning angle of the robot in this real experiment are $3.90 \mathrm{~m}$ and $433.30^{\circ}$, respectively. The results of this experiment show that the effectiveness and efficiency of the proposed approach in real environments.

\section{Conclusion}

The memetic algorithm-based path planning approach of mobile robot is investigated in this paper. To deal with the shortcomings of the general memetic algorithm in the path planning task, an improved memetic algorithm with variable length chromosome is proposed, where both the global search and the local search process are improved. In the global process of the memetic algorithm, an improved two-point crossover and a bacterial mutation are used to improve the convergence of the general GA in the proposed approach. In the local search process, a disorder strategy and neighbouring search are used to improve the search efficiency of the memetic algorithm. The path planning task is achieved efficiently based on the proposed approach under various situations, such as the environment is large and the obstacles are linked with difficult shapes. In future work, some new environment modelling methods and novel bio-inspired methods will be studied in the path planning approach for robot.

\section{Acknowledgement}

This work was supported by the National Natural Science Foundation of China (61203365, 61573128), the Jiangsu Province Natural Science Foundation (BK2012149) and the Fundamental Research Funds for the Central Universities (2015B20114).

\section{References}

[1] T.-K. Wang, Q. Dang, and P.-Y. Pan, Path planning approach in unknown environment, International Journal of Automation and Computing, 7(3), 2010, 310-316.

[2] M.A.K. Jaradat, M.H. Garibeh, and E.A. Feilat, Autonomous mobile robot dynamic motion planning using hybrid fuzzy potential field, Soft Computing, 16(1), 2012, 153-164.

[3] E. Galceran and M. Carreras, A survey on coverage path planning for robotics, Robotics and Autonomous Systems, 61(12), 2013, 1258-1276.

[4] L. Deng, X. Ma, J. Gu, Y. Li, Z. Xu, and Y. Wang, Artificial immune network-based multi-robot formation path planning with obstacle avoidance, International Journal of Robotics and Automation, 31(3), 2016, 233-242.

[5] S. Ge and Y. Cui, Dynamic motion planning for mobile robots using potential field method, Autonomous Robots, 13(3), 2002, $207-222$.

[6] T.-K. Lee, S.-H. Baek, Y.-H. Choi, and S.-Y. Oh, Smooth coverage path planning and control of mobile robots based on high-resolution grid map representation, Robotics and Autonomous Systems, 59(10), 2011, 801-812.
[7] J. Kim, M. Kim, and D. Kim, Variants of the quantized visibility graph for efficient path planning, Advanced Robotics, 25(18), 2011, 2341-2360.

[8] J. Ni, L. Wu, X. Fan, and S.X. Yang, Bioinspired intelligent algorithm and its applications for mobile robot control: A survey, Computational Intelligence $\&$ Neuroscience, 2016(2), 2016, 1-16.

[9] J. Chen, S. Xie, H. Li, J. Luo, and K. Feng, Robot path planning based on adaptive integrating of genetic and ant colony algorithm, International Journal of Innovative Computing, Information and Control, 11(3), 2015, 833-850.

[10] D. Liu, L. Wang, and K. Tan, Design and control of intelligent robotic systems (Heidelberg: Springer, 2009).

[11] O. Montiel, U. Orozco-Rosas, and R. Sepulveda, Path planning for mobile robots using bacterial potential field for avoiding static and dynamic obstacles, Expert Systems with Applications, 42(12), 2015, 5177-5191.

[12] J. Ni, X. Li, M. Hua, and S.X. Yang, Bioinspired neural network-based Q-learning approach for robot path planning in unknown environments, International Journal of Robotics and Automation, 31(6), 2016, 464-474.

[13] Y. Xue and H. Liu, Optimal path planning in complex indoor environment based on improved PSO, Journal of Computational Information Systems, 7(6), 2011, 2158-2165.

[14] P. He and S. Dai, Real-time stealth corridor path planning for fleets of unmanned aerial vehicles in low-altitude penetration, International Journal of Robotics and Automation, 30(1), 2015, $60-69$

[15] J. Botzheim, Y. Toda, and N. Kubota, Bacterial memetic algorithm for offline path planning of mobile robots, Memetic Computing, 4(1), 2012, 73-86.

[16] Z. Zhu, F. Wang, S. He, and Y. Sun, Global path planning of mobile robots using a memetic algorithm, International Journal of Systems Science, 46(11), 2015, 1982-1993.

[17] G. Fraser, A. Arcuri, and P. McMinn, A memetic algorithm for whole test suite generation, Journal of Systems and Software, 103(C), 2015, 311-327.

[18] H. Qu, K. Xing, and T. Alexander, An improved genetic algorithm with co-evolutionary strategy for global path planning of multiple mobile robots, Neurocomputing, 120, 2013, 509-517.

[19] P.-C. Chang, S.-S. Chen, and C.-Y. Fan, Mining gene structures to inject artificial chromosomes for genetic algorithm in single machine scheduling problems, Applied Soft Computing Journal, 8(1), 2008, 767-777.

[20] P. Foldesi and J. Botzheim, Modeling of loss aversion in solving fuzzy road transport traveling salesman problem using eugenic bacterial memetic algorithm, Memetic Computing, 2(4), 2010, 259-271.

[21] A. Prugel-Bennett, Modeling crossover-induced linkage in genetic algorithms, IEEE Transactions on Evolutionary Computation, 5(4), 2001, 376-387.

[22] G.E. Jan, K.Y. Chang, and I. Parberry, Optimal path planning for mobile robot navigation, IEEE/ASME Transactions on Mechatronics, 13(4), 2008, 451-460.

[23] P. Raja and S. Pugazhenthi, On-line path planning for mobile robots in dynamic environments, Neural Network World, 22(1), 2012, 67-83.

[24] Y. Gao and S.-D. Sun, Local path planning of mobile robots in dynamic unknown environment based on prediction of collision, 2009 Int. Conf. Measuring Technology and Mechatronics Automation, ICMTMA 2009, vol. 2, Zhangjiajie, Hunan, China, 2009, 84-88.

[25] Y. Wang, I.P.W. Sillitoe, and D.J. Mulvaney, Mobile robot path planning in dynamic environments, Proceedings - IEEE Int. Conf. Robotics and Automation, Rome, Italy, 2007, 71-76.

[26] L. Sun, Y. Luo, X. Ding, and L. Wu, Path planning and obstacle avoidance for mobile robots in a dynamic environment, Open Automation and Control Systems Journal, 6(1), 2014, $77-83$. 


\section{Biographies}

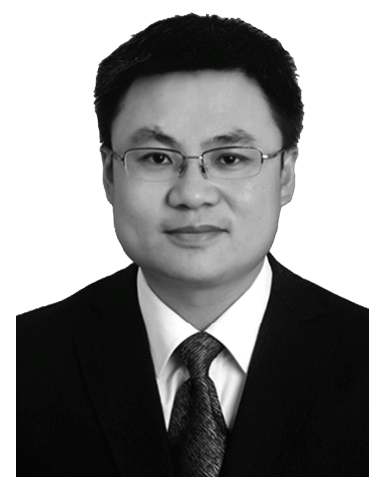

Jianjun $N i$ received his Ph.D. degree in Control Theory and Control Engineering from China University of Mining and Technology, Xuzhou, China, in 2005. He was a Visiting Professor with the Advanced Robotics \& Intelligent Systems (ARIS) Laboratory at the University of Guelph in Canada from November 2009 to October 2010. He is currently a Professor of College of IOT Engineering at Hohai University, China. His research interests include fuzzy systems, neural networks, robotics, machine intelligence and multi-agent system.

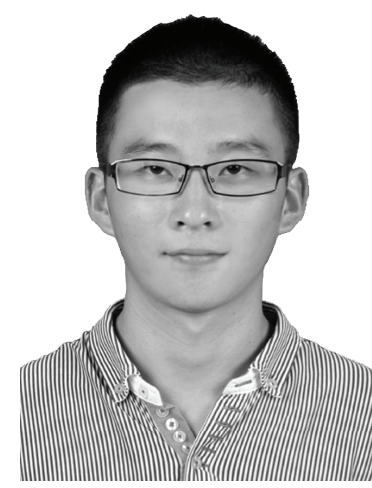

Kang Wang received the B.S. degree in Automation from Hohai University, China, in 2015. Currently, he is working towards the M.S. degree in the College of IOT Engineering, Hohai University. His research interests include robotic path planning and simultaneous localization and mapping.

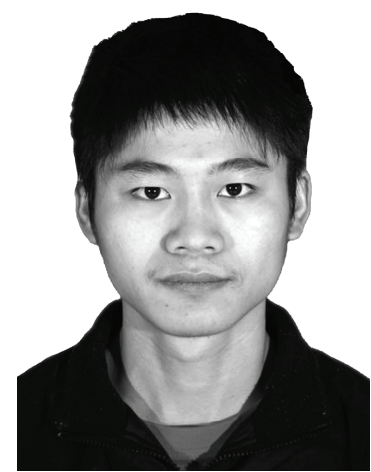

Qingyun Cao received the B.S. degree in Telecommunication Engineering from Hohai University, China, in 2013. Currently, he is working towards the M.S. degree in the College of IOT Engineering, Hohai University, China. His research interests include robot navigation, bio-inspired intelligence and machine learning.

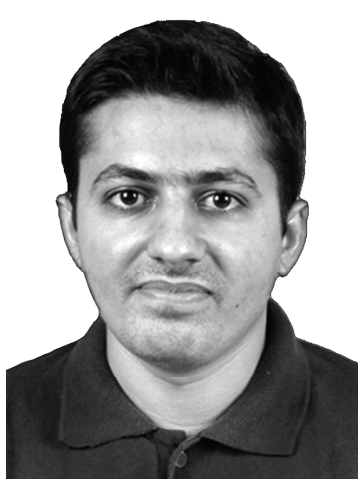

Zubair Khan received the B.S. degree in Telecommunication Engineering from The Islamia University of Bahawalpur, Pakistan, in 2012. Currently, he is working towards the M.S. degree in the College of IOT Engineering, Hohai University, China. His research interests include digital image processing and robot control.

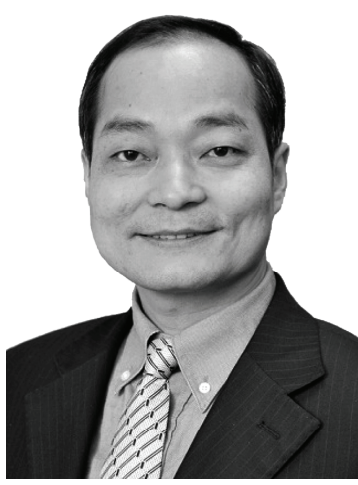

Xinnan Fan received the Ph.D. degree in Hydroinformatics from Hohai University, Nanjing, China, in 2009. Currently, he is a Professor of the College of IOT Engineering, Hohai University, China. His research interests include robotics, image processing, and automation. 